\title{
Magnetic-gateable valley exciton emission
}

\author{
Helena Bragança $\mathbb{D}^{1,2}$, Hao Zeng $\mathbb{D}^{3 凶}$, Alexandre Cavalheiro Dias ${ }^{1}$, Jorge Huamani Correa ${ }^{1}$ and Fanyao Qu $\mathbb{D}^{1 凶}$
}

The use of valley excitonic states of transition metal dichalcogenides to store and manipulate information is hampered by fast carrier recombination and short valley lifetime. We propose theoretically a scheme to overcome such an obstacle, by applying a tilted exchange field through the magnetic proximity effect on monolayer $\mathrm{MoS}_{2}$. While the in-plane component of the exchange field brightens the dark exciton by spin mixing, the out-of-plane field can effectively gate the emission with an ON/OFF ratio of 2700. Importantly, the brightening is valley selective, leading to nearly $100 \%$ valley and spin polarization at room temperature. The resulting strongly gateable dark-exciton emission with long lifetime and near unity valley polarization makes it convenient to manipulate the valley degree of freedom, which may offer new paradigm for information processing and transmission.

npj Computational Materials (2020)6:90; https://doi.org/10.1038/s41524-020-00356-w

\section{INTRODUCTION}

Monolayer transition metal dichalcogenides (TMDs), namely $\mathrm{MX}_{2}$ $(\mathrm{M}=\mathrm{Mo}, \mathrm{W} ; \mathrm{X}=\mathrm{S}$, Se, Te), have attracted great interest for information technology. They are direct gap semiconductors with two inequivalent valleys located at the $\mathrm{K}$ and $\mathrm{K}^{\prime}$ points of the hexagonal Brillouin zone ${ }^{1-6}$. Owing to the 2D spatial confinement and reduced dielectric screening, TMDs exhibit strong Coulomb interactions 7,8 . Upon light excitation, it favors the formation of tightly bound electron-hole pairs called excitons ${ }^{9}$. Because of their high binding energies of up to a few hundred meV, these excitons are stable even at room temperature ${ }^{10-14}$. Due to the large spin-orbit coupling (SOC), monolayer TMDs possess spinsplit in the range of hundreds of $\mathrm{meV}^{15}$ in the top valence band (VB). The bottom of the conduction band (CB) is also spin-split. However, it is an order of magnitude smaller than the former ${ }^{16}$, being comparable to the room-temperature thermal energy. Such band spin splittings give rise to optically active and inactive interband transitions from the top-most VB to the two spin-split $\mathrm{CBs}$, resulting in bright and dark excitons ${ }^{17}$. The former with antiparallel electron and hole spins (i.e., parallel electron spins in VB and $\mathrm{CB}$ ) carry total angular momentum of $|M|=1$, allowing the bright states to couple selectively to light with right-handed (lefthanded) circular polarization in the $\mathrm{K}\left(\mathrm{K}^{\prime}\right)$ valley ${ }^{18}$. However, both their radiative lifetime and valley lifetime are very short due to the large oscillator strength and strong long-range electron-hole exchange interaction ${ }^{19-22}$, undermining their potential for applications. In contrast, the spin-forbidden dark excitons with parallel electron and hole spins (i.e., antiparallel electron spins in VB and CB) have total angular momentum $|M|=2$. This spin mismatch strongly suppresses their radiative recombination, thus the dark excitons possess long lifetime of the order of $\mathrm{ns}^{23}$. In addition, the intervalley scattering of dark states is weak due to the spin flip, leading to long valley lifetime ${ }^{24}$. Such long radiation and valley lifetimes make dark excitons attractive for optically controlled information processing. Nevertheless, their optical inactivity poses a significant challenge for experiments. Although an in-plane magnetic field has recently been employed to brighten the dark excitons, the field magnitude required for moderate brightening is as high as few ten tesla ${ }^{25,26}$, making practical applications difficult. Besides, the lack of valley selectivity poses another challenge to manipulating the valley exciton states ${ }^{27-31}$. These obstacles need to be overcome to make practical applications feasible.

In this work, we propose a theoretical scheme to brighten the dark excitons in monolayer $\mathrm{MoS}_{2}$ with an efficiency far exceeding that by an in-plane magnetic field. Our scheme relies on coupling of the monolayer $\mathrm{MoS}_{2}$ to a magnetic substrate which supplies a tilted exchange field $d^{32-36}$. We show that the in-plane $\left(J_{\|}\right)$, and outof-plane $\left(J_{\perp}\right)$, components of the exchange field work synergistically for dark-exciton brightening. While it is known that an inplane exchange field is the origin of spin mixing and dark-exciton brightening $^{37}$, out-of-plane exchange field shifts the two spin-split $\mathrm{CBs}$ in opposite directions. Such a shift reduces the CB spin gap in one of the two valleys, which favors the spin mixing. Large $J_{\perp}$ can even lead to CB level crossing (Fig. 1a) that switches the characteristics of the exciton ground state from bright to dark. This increases the dark state population and its emission intensity, as shown schematically in Fig. $1 \mathrm{~b}$. In the other valley, however, it enlarges the gap and suppresses the spin mixing. Thus, the out-ofplane exchange field acts as a gate to control the on and off states of dark-exciton emission. Recently, an exciton transistor with electric gating has been reported ${ }^{38}$. Relying on magnetic gating, our scheme can achieve very large ON/OFF ratio without concern of dielectric breakdown. Therefore, this valley-selective gating of dark-exciton emission with long lifetime and near unity valley polarization (VP) at room temperature represents a step forward for applications of valley excitons in valleytronics and optoelectronics.

\section{RESULTS}

Single-particle band structure

To reveal the mechanism of the transistor-like behavior of valley excitons, we start by discussing the effects of an out-of-plane exchange field on the single-particle band structure of $\mathrm{MoS}_{2}$. For this, we combine density functional theory calculation with an 11 bands tight-binding model that consider the effective exchange field supplied by a ferromagnetic substrate (see the "Methods" section for details about the single-particle calculation). The left panel in Fig. 1a shows the behavior of the conduction bands in the $\mathrm{K}$ valley. In the absence of exchange, the spin-up band, $C B_{\uparrow}$, is the

${ }^{1}$ Instituto de Física, Universidade de Brasília, Brasília-DF 70919-970, Brazil. ²Departamento de Física, Universidade Federal de Ouro Preto, Ouro Preto, Minas Gerais 35400-000, Brazil. ${ }^{3}$ Department of Physics, University at Buffalo, the State University of New York, Buffalo, NY 14260, USA. ${ }^{\circledR}$ email: haozeng@buffalo.edu; fanyao@unb.br 


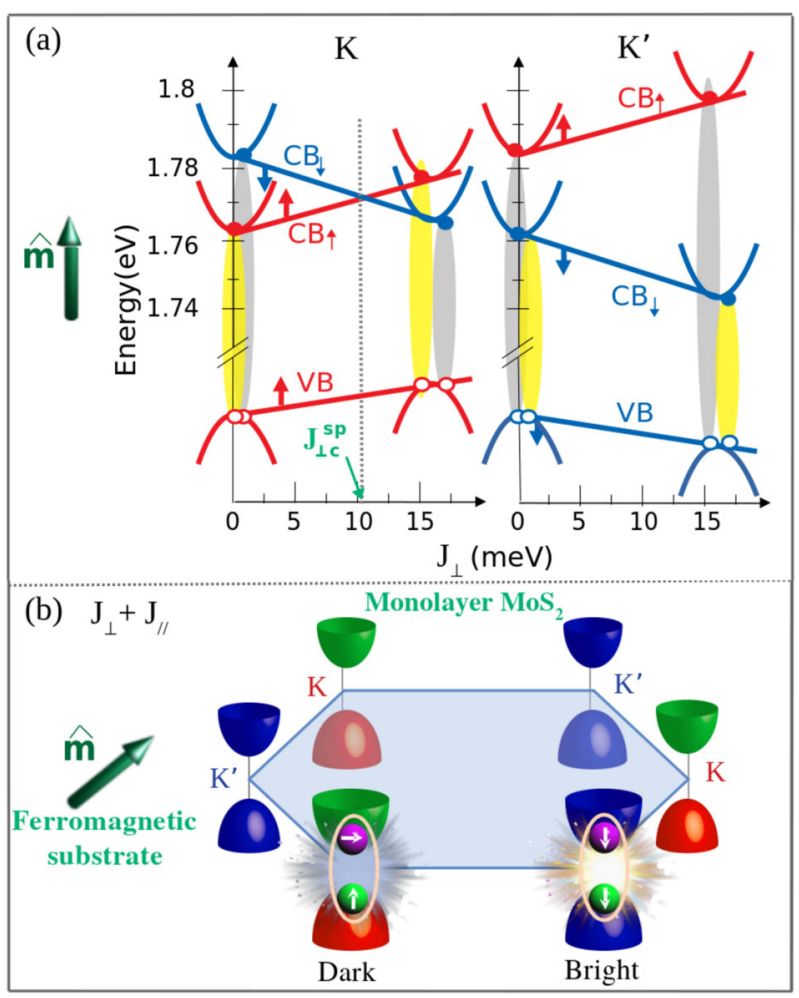

Fig. 1 Magnetic proximity effect on excitons in a monolayer $\mathrm{MoS}_{2}$ deposited on a ferromagnetic substrate. a Energies of minimum CBs and maximum VB at K (left) and $K^{\prime}$ (right) valleys as a function of out-of-plane exchange field (substrate magnetization $\hat{m}$ in the $\hat{z}$ direction). Red and blue lines indicate spin-up and -down bands, respectively. The Zeeman shift in the $\mathrm{CBs}$ reduces the spin gap in the $\mathrm{K}$ valley, and leads to band crossing at $J_{\perp}=J_{\perp c}^{s p}$. Consequently, the exciton ground state switches from bright (shown by yellow) to dark (shown by gray). In the $\mathrm{K}^{\prime}$ valley, on the other hand, the CB spin gap increases with increasing $J_{\perp}$. b The first Brillouin zone and simplified band dispersion around $\mathrm{K}$ and $\mathrm{K}^{\prime}$ valleys. Blue, red, and green colors represent spin-down, spin-up, and mixed spin states of electronic bands, respectively. Under a tilted exchange field, the CB spin mixing is stronger in $\mathrm{K}$ than in $\mathrm{K}^{\prime}$ valley, due to the smaller spin gap. This leads to a valley-selective dark-exciton brightening. The exciton ground states are shown, which are bright in $\mathrm{K}^{\prime}$ valley and brightened-dark in $\mathrm{K}$ valley.

ground state, separated from the spin-down band, $\mathrm{CB}_{\downarrow}$, due to the SOC, $\Delta_{C}=20.7 \mathrm{meV}$. Applying an out-of-plane exchange field $J_{\perp}$ shifts the energy level of $\mathrm{CB}_{\uparrow}$ up and that of $\mathrm{CB}_{\downarrow}$ down, decreasing the spin gap in the $\mathrm{K}$ valley. The spin gap between $\mathrm{CB}_{\uparrow}$ and $\mathrm{CB}_{\downarrow}$ is proportional to $\left|J_{\perp}-\Delta_{C} / 2\right|$, i.e., it increases with lowering $J_{\perp}$ for $J_{\perp}<10 \mathrm{meV}$ and with increasing $J_{\perp}$ for $J_{\perp}>10 \mathrm{meV}$ (see Methods section for an effective Hamiltonian for singleparticle band energies). We have then identified the critical out-ofplane exchange field, $J_{\perp c}^{s p} \approx 10 \mathrm{meV}$, for which the CB levels cross in the $\mathrm{K}$ valley. At $\mathrm{K}^{\prime}$ point (Fig. 1a, right panel), the $C B$ spin gap increases monotonically with increasing $J_{\perp}$.

Let us now consider a tilted exchange field, with both out-ofplane and in-plane components, $J_{\|}$and $J_{\perp}$. Figure 2 shows the $C B$ energies as a function of $J_{\|}$for $J_{\perp}=0,2$, and $11 \mathrm{meV}$, in the $\mathrm{K}$ (top) and $\mathrm{K}^{\prime}$ (bottom) points, respectively. The two non-zero $J_{\perp}$ values were judiciously chosen so that the former is smaller while the latter is larger than the critical field $J_{\perp c}^{s p}$ for CB level crossing in the $\mathrm{K}$ valley. Moreover, $J_{\perp}=11 \mathrm{meV}$ is much closer in energy to $J_{\perp c}^{s p}$ than $J_{\perp}=2 \mathrm{meV}$. It can be seen that while spin mixing is achieved by applying a $J_{\|}$, the degree of spin mixing is sensitively controlled by $J_{\perp}$. In Fig. 2, the degree of spin mixing is represented by the $z$-component of the spin expectation value of the $C B$ electrons,
$\left\langle\hat{S}_{z}\right\rangle$, shown by the colors and arrows. The initially spin-up (red) and spin-down (blue) $\mathrm{CBs}$ at $J_{\perp}=0$ are labeled as $\mathrm{CB}_{\uparrow}$ and $\mathrm{CB}_{\downarrow}$, respectively. To show all bands involved in the first optical transitions, we also plot the energy of the highest VB. For $J_{\perp}=0$, the $C B_{\uparrow}$ level is lower (higher) than the $C B_{\downarrow}$ in $K\left(K^{\prime}\right)$ valley by $\Delta_{c}=20.7 \mathrm{meV}$, as seen in Fig. 2a and d. Since the lowest CB and the highest VB have the same spin orientation, the optical transition between them is spin allowed (bright exciton states); while that between the second lowest $C B$ and the top VB is spin forbidden (dark-exciton states). With increasing $J_{\|}$, two effects are observed: (I) there is a progressive spin tilting, i.e., spin mixing in the two CB branches, as indicated by the color evolution of the bands and tilting of the spin arrows; (II) the energy of the lowest $C B$ decreases, and that of the second lowest $C B$ increases; while the energy of the top VB always increases. This behavior is the same for both valleys. The shift is roughly an order of magnitude smaller than the Zeeman shift due to $J_{\perp}$ (Fig. 1a), and is attributed to the renormalization of the band energy ${ }^{26,39}$. It enlarges the gap between the CBs, which decreases the degree of spin mixing than that without the renormalization. We also note that VB does not show a spin tilt as a function of $J_{\|}$in either $\mathrm{K}$ or $\mathrm{K}^{\prime}$ valley. This is because the spin-orbit splitting for the top VBs is of the order of $400 \mathrm{meV}$; such large energy separation prevents spin mixing under an exchange field.

For $J_{\perp}=2 \mathrm{meV}$, the two CB levels become closer in the $\mathrm{K}$ valley (see Fig. 2b), while the $C B$ gap increases in the $K^{\prime}$ valley (Fig. 2e). This enhances the spin mixing in the former while reduces it in the latter. For example, at $J_{\|}=30 \mathrm{meV}$, the spin tilting angle increases to $74^{\circ}$ in the $\mathrm{K}$ valley while decreases to $67^{\circ}$ in the $\mathrm{K}^{\prime}$ valley, from $71^{\circ}$ for both valleys with $J_{\perp}=0$. With further increasing $J_{\perp}$ to $11 \mathrm{meV}, \mathrm{CB}$ level crossing occurs in the $\mathrm{K}$ valley (Fig. $2 \mathrm{C}$ ); i.e., $\mathrm{CB}_{\uparrow}$ energy is now higher than $C_{\downarrow}$, in contrast to the cases in panels (a) and (b). At $J_{\|}=0$, the two CBs in the $K$ valley becomes nearly degenerate. In comparison with the case for $J_{\perp}=2 \mathrm{meV}$ (far away from $J_{\perp c}^{s p}$ the spin tilt in $\mathrm{K}$ valley produced by $J_{\|}$is far more efficient. For example, at a small $J_{\|}$of $5 \mathrm{meV}$, the spin tilting angle already reaches $82^{\circ}$, which is more than three times higher than the value of $26^{\circ}$ without $J_{\perp}$. In $\mathrm{K}^{\prime}$ valley (Fig. 2f), on the other hand, the $C B$ gap is further enlarged and $J_{\|}$hardly tilts the $C B$ spins.

Therefore, the origin of $C B$ spin mixing is the in-plane component of the exchange field, $J_{\|}$. However, at $J_{\perp}=0$, such a mixing is not efficient due to the large spin gap (of $\sim 20 \mathrm{meV}$ ) in the CBs of $\mathrm{MoS}_{2}$. Applying an out-of-plane exchange field can reduce the spin gap and even switch the order of the $C B s$ in $K$ valley, which intensifies the spin mixing. Close to $J_{\perp c}^{s p}$ for $C B$ crossing, the spin mixing is the most efficient. Thus $J_{\perp}$ can strongly modulate the degree of spin mixing. The contrasting behavior in $\mathrm{K}$ and $\mathrm{K}^{\prime}$ valleys causes valley-selective brightening of the originally spin-forbidden dark exciton, as will be discussed later.

To further explore the valley-selective band crossing and spin tilting, Fig. 3 shows a contour plot of the expectation values of the $z$-component of electron spins $\left\langle\hat{S}_{z}\right\rangle$ in $\mathrm{CBs}$ at $\mathrm{K}$ and $\mathrm{K}^{\prime}$ points as functions of in-plane $\left(J_{\|}\right)$and out-of-plane $\left(J_{\perp}\right)$ components of the exchange field. The value of $\left\langle\hat{S}_{z}\right\rangle$ is represented by the color scale. We start our analysis from $C B_{\uparrow}$ and $C B_{\downarrow}$ bands in the $K$ valley, shown in Fig. 3a and $C$, respectively. As pointed out earlier, $J_{\perp}$ tunes the spin gap, with a crossing of $\mathrm{CB}_{\uparrow}$ and $C B_{\downarrow}$ at $J_{\perp}=J_{\perp C^{\prime}}^{S D^{\prime}}$ shown by the horizontal black dashed line. Application of a $J_{\|}$, on the other hand, leads to mixing of spin-up and spin-down states. The effectiveness of mixing is dependent on the energy separation between these two states, which can be tuned by $J_{\perp}$. The maximum spin mixture $\left(\left\langle\hat{S}_{z}\right\rangle=0\right)$ occurs at the crossing point, i.e., at $J_{\perp}=J_{\perp c^{\prime}}^{s p}$ where $\mathrm{CB}_{\uparrow}$ and $\mathrm{CB}_{\downarrow}$ are degenerate. With increasing $C B$ spin gap by either decreasing or increasing $J_{\perp}$, mixing becomes less efficient; as a result, higher $J_{\|}$is needed to obtain the same degree of spin mixing. Quantitatively, $J_{\perp}$ depends on $J_{\|}$for constant values of $\left\langle S_{z}\right\rangle$. This relationship is described by $J_{\perp}=a J_{\|}+b$, where the slope $a=\left\langle S_{z}\right\rangle\left(-\left\langle S_{z}\right\rangle\right)$ in the upper (lower) 


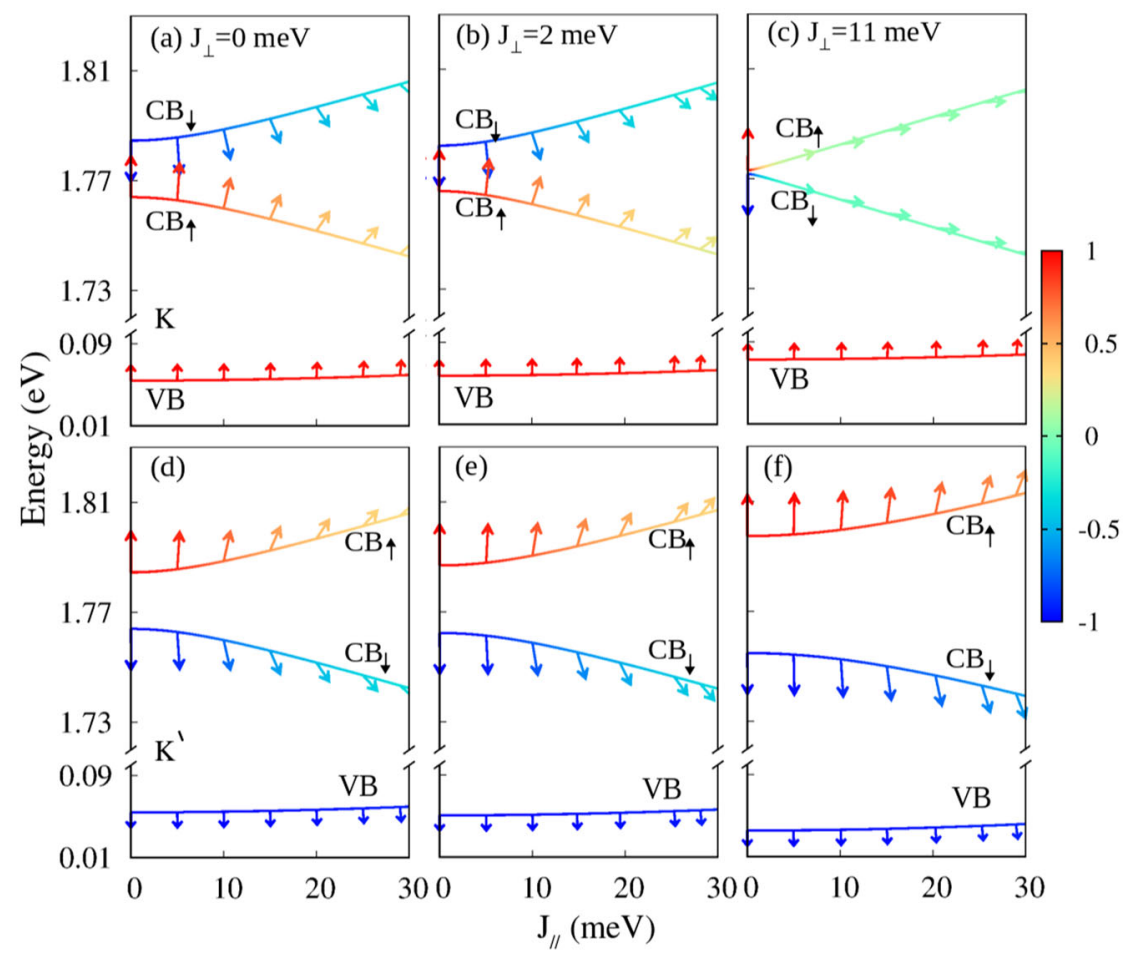

Fig. 2 Single-particle band energy as a function of exchange field. Evolution of the two lowest CBs and the highest VB as $J_{\|}$increases for $J_{\perp}=0 \mathrm{meV}(\mathbf{a}, \mathbf{d}), J_{\perp}=2 \mathrm{meV}(\mathbf{b}, \mathbf{e})$, and $J_{\perp}=11 \mathrm{meV}(\mathbf{c}, \mathbf{f})$ at $\mathrm{K}$ (top) and $\mathrm{K}^{\prime}$ (bottom) points. $\mathrm{CB}_{\uparrow}$ and $\mathrm{CB}_{\downarrow}$ label the initially spin-up and initially spin-down CB states, respectively. The spin mixing is reflected by the color (values displayed on the colorbar in units of $\hbar / 2$ ) and also by arrows that indicate the spin direction.

$\mathrm{CB}$, and $b=\Delta_{C} / 2 \sim 10 \mathrm{meV}$ (see Supplemental Sec. II and Supplementary Figure 2 for an analytical derivation of this relation). This analytical results is in perfect agreement with numerical tight-binding data, as shown in Fig. 3. When the fixed values of $\left\langle S_{z}\right\rangle$ ranging from -1 to +1 in step size of 0.25 are plotted in dashed lines, a fan shape is observed. In addition, the behavior of $\mathrm{CB}_{\uparrow}$ and $\mathrm{CB}_{\downarrow}$ are symmetric, only with opposite spin polarity. Let us now turn our attention to $K^{\prime}$ valley, where the spin orientation is opposite to that in $\mathrm{K}$ valley due to time reversal symmetry. Increasing $J_{\perp}$ always enlarges spin gap monotonically ${ }^{40}$, making the spin mixing less efficient. Only at low $J_{\perp}$ and very large $J_{\|}$, there is moderate spin tilting in $\mathrm{K}^{\prime}$ valley, as shown in Fig. $3 b$ and $d$. The contrasting behavior in $K$ and $K^{\prime}$ valleys causes valley-selective brightening of the originally spin-forbidden dark exciton, as discussed in the following.

\section{Exciton emission and valley polarization}

Knowing the influence of the magnetic proximity effect on the single-particle-band structure and spin mixing, we now turn our attention to the impact of the exchange field on the exciton emission and VP, derived by solving Bethe Salpeter exciton equation and rate equations for exciton population (see Methods section). In the $\mathrm{K}$ valley, the bright (dark) exciton $X_{b}\left(X_{d}\right)$ is formed by a hole in the top $\mathrm{VB}$ and an electron in $\mathrm{CB}_{\uparrow}\left(\mathrm{CB}_{\downarrow}\right)$. In the top panels of Fig. 4 we show the room-temperature photoluminescence (PL) intensity of initially bright, $X_{b}$, and initially dark, $X_{d}$ excitons in the $K$ valley, as a function of the in-plane exchange field, $J_{\|}$, for different values of $J_{\perp}$. At $J_{\|}=0$ the optical selection rules ensure that $X_{b}$ is optically active, while $X_{d}$ does not couple with light. When $J_{\|}$increases, however, $X_{d}$ starts to emit light, with the efficiency of brightening being strongly tuned by $J_{\perp}$. This is akin to the carrier density and thus source-drain current in a transistor tuned by a gate voltage. At $J_{\perp}=0$ (Fig. 4a), with increasing $J_{\|}$, the emission intensity from $X_{d}$ first increases, reaches a maximum, and then decreases with further increasing $J_{\|}$. The brightening of the initially dark exciton is due to the $C B$ spin mixing, making optical transition allowed for the initially dark exciton. The non-monotonic behavior of PL intensity with increasing $J_{\|}$is attributed to the competition between spin mixing and the renormalization of the band gap. As can be seen in Fig. 2, the energy gap between the two CBs increases with increasing $J_{\|}$, which in turn increases the energy separation between the two intravalley exciton states, reducing the exciton population of the excited (originally dark) state at large $J_{\|}$. In contrast, the $J_{\|}$ dependence of bright exciton, $X_{b}$, shows an opposite trend to that of $X_{d}$. Namely, while $X_{d}$ exhibits a maximum, $X_{b}$ shows a minimum. With increasing $J_{\perp}$, the bright-dark-exciton gap shrinks, making thermal population of the excited state more effective at room temperature. The dark-exciton brightening thus becomes more efficient at the same $J_{\| \cdot}$. This is manifested in the plot of $X_{d}$ intensity for $J_{\perp}=5 \mathrm{meV}$ (Fig. $4 \mathrm{~b}$ ), where the maximum PL quantum yield increases to $22 \%$ from $7 \%$ at $J_{\perp}=0$. Moreover, the $J_{\|}$at which PL showing maximum decreases to $2.6 \mathrm{meV}$ from $10 \mathrm{meV}$, suggesting that dark-exciton brightening by applying a titled exchange field is far more effective than applying an inplane field.

For a larger $J_{\perp}=15 \mathrm{meV}$ (Fig. 4c), the ground state switches into the dark state in $\mathrm{K}$ valley, while it remains bright in $\mathrm{K}^{\prime}$ one. Note that although the $\mathrm{CB}$ crossing happens at $J_{\perp} \sim 10 \mathrm{meV}$, the exciton ground state switches from bright to dark at a smaller out-of-plane field, $J_{\perp c}^{e x} \sim 5.1 \mathrm{meV}$; that is, $J_{\perp c}^{e x}<J_{\perp c}^{s p}$. This is because the brightdark-exciton separation is smaller than the spin-orbit splitting, as the dark exciton has larger binding energy than the bright one. As can be seen in Fig. 4c, the PL intensity of the initially dark exciton increases monotonically with increasing $J_{\|}$. This is due to the combination of the following two effects: (I) the in-plane exchange field increases the spin mixing, enhancing the coupling of the state with light; (II) $X_{d}$ is the ground state and its energy decreases with increasing $J_{\|}$. The large bright-dark energy separation at 


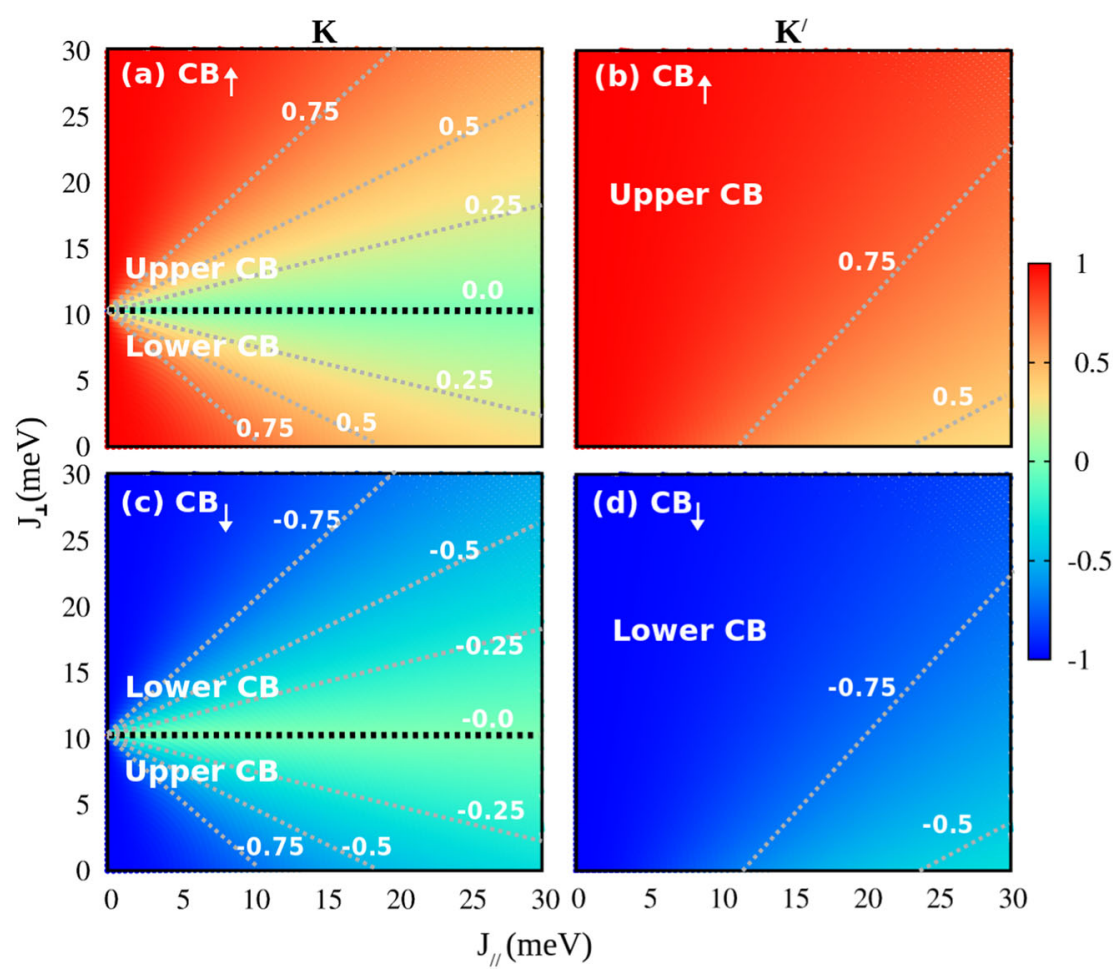

Fig. 3 Spin mean value of the conduction bands. Spin-splitted CBs at $\mathrm{K}$ (left panels) and $\mathrm{K}^{\prime}$ (right panels) points of monolayer MoS ${ }_{2}$ on ferromagnetic substrate. The color code represents the mean value of the spin in $z$ direction, $\left\langle S_{z}\right\rangle$, for $C B_{\uparrow}(\mathbf{a}, \mathbf{b})$ and $C B_{\downarrow}(\mathbf{c}, \mathbf{d})$ as functions of the out-of-plane $\left(J_{\perp}\right)$ and in-plane $\left(J_{\|}\right)$components of exchange field. In K valley there is a CB crossing as a function of $J_{\perp}$ : CB $B_{\uparrow}$ correspond to the lower $\mathrm{CB}$ for $J_{\perp}<10 \mathrm{meV}$ and the upper $\mathrm{CB}$ otherwise. In $\mathrm{K}^{\prime}$ valley, on the other hand, $\mathrm{CB}_{\uparrow}$ has always higher energy than $\mathrm{CB}_{\downarrow}$. $\mathrm{Gray}$ dotted lines correspond to fixed values of $\left\langle S_{z}\right\rangle$ indicated by numbers (in units of $\hbar / 2$ ).

larger $J_{\|}$reduces the thermal population of the excited state $X_{b}$, which enhances $X_{d}$ emission.

In the $\mathrm{K}^{\prime}$ valley, the dark-exciton brightening effect is negligible due to the large bright-dark energy separation, which is further increased by $J_{\perp}$. Besides the small oscillator strength of the dark state $\left(X_{d}^{\prime}\right)$, it has lower population because it is always the excited state. Both effects make the PL intensity of $X_{d}^{\prime}$ to be vanishingly small irrespective of the values of $J_{\perp}$ and $J_{\|}$. Concomitantly, the bright state in the $\mathrm{K}^{\prime}$ valley, $X_{b}^{\prime}$, is only slightly darkened. The negligible brightening effect in the $\mathrm{K}^{\prime}$ valley under a tilted exchange field and the valley-protected $X_{b}^{\prime}$ emission is show in Supplementary Figure 5 of the Supplemental material. Importantly, the valley-selective dark-exciton brightening causes strong darkexciton valley polarization. The valley polarization of bright and dark excitons, are defined as $V P_{j}=\left[I\left(X_{j}\right)-I\left(X_{j}^{\prime}\right)\right] /\left[I\left(X_{j}\right)+I\left(X_{j}^{\prime}\right)\right]$, where $I\left(X_{j}\right)$ represents the PL intensity of the $X_{j}$ state, with $j=\{b, d\}$, and (') labels states in the $K^{\prime}$ valley. In Fig. $4 \mathrm{~d}-\mathrm{f}$, we show room temperature $V P_{b}$ and $V P_{d}$ as a function of $J_{\|}$, for different values of $J_{\perp}$. For $J_{\perp}=0$, time reversal symmetry is preserved, therefore both $V P_{b}$ and $V P_{d}$ is null for all values of $J_{\|}$. For $J_{\perp} \neq 0$, on the other hand, we observe that $V P_{b}$ is strongly tuned by the exchange field. For $J_{\perp}=5 \mathrm{meV}$ (Fig. 4e) the bright exciton is the ground state in both valleys. $V P_{b}$ is initially positive (stronger emission from $\mathrm{K}$ valley) due to the valley splitting produced by $J_{\perp}$, namely, the states in $\mathrm{K}^{\prime}$ valley have higher energy than that in $\mathrm{K}$ valley, which enhances the intervalley scattering from $\mathrm{K}^{\prime}$ to $\mathrm{K}$ valley (see Methods section for the description of intervalley scattering). $V P_{b}$ shows a nonmonotonic behavior that follows the non-monotonic dependence of $I\left(X_{b}\right)$ on $J_{\|}$(Fig. $\left.4 b\right)$ : it first decreases from 12 to $-10.5 \%$ due to the valley-selective darkening of the $X_{b}$ state, then increases when $I\left(X_{b}\right)$ further increases due to band renormalization, and reaches $23 \%$ at $J_{\|}=30 \mathrm{meV}$. For larger $J_{\perp}=15 \mathrm{meV}$ (Fig. $4 \mathrm{f}$ ), $V P_{b}$ goes from positive $\left(45 \%\right.$ at $\left.J_{\|}=0\right)$ to negative values $\left(-32 \%\right.$ at $\left.J_{\|}=30 \mathrm{meV}\right)$ monotonically with increasing $J_{\|}$. This is due to combined effects of the valley-selective darkening of $X_{b}$ and enhanced population of $X_{b}^{\prime}$ state due to increasing bright-dark separation in $K^{\prime}$ valley with increasing $J_{\|}$. Compared with bright excitons, the $X_{d}$ emission exhibits significantly more robust VP. For $J_{\perp}=5 \mathrm{meV}, V P_{d}$ quickly reaches almost $100 \%$ at $J_{\|}=0.1 \mathrm{smeV}$, due to valley-selective brightening. Although it slowly decreases with increasing $J_{\|,}$ following the decrease of $X_{d} \mathrm{PL}$ intensity, $V P_{d}$ remains larger than $80 \%$ even at $J_{\|}=30 \mathrm{meV}$. With increasing $J_{\perp}$ to $15 \mathrm{meV}, V P_{d}$ holds a constant value close to $100 \%$ irrespective of $J_{\|}$. The robust VP close to unity can be explained by the combined effects of valleyselective spin mixing and enhanced $X_{d}$ population due to valleyselective switching of exciton ground state from $X_{b}$ to $X_{d}$ upon application of a large $J_{\perp}$.

To further compare the effect of dark-exciton brightening by a tilted exchange field with that by an in-plane one, we show in Fig. 5 the evolution of the PL spectra as a function of the total exchange field for (a) $\theta=90^{\circ}$ (in-plane) and (b) $\theta=30^{\circ}$ (tilted). Brightened-dark-exciton emission is observed in both cases. The dark-exciton emission is barely visible for the case of $\theta=90^{\circ}$. Remarkably, for $\theta=30^{\circ}$, the intensity of the brightened-dark exciton is dramatically enhanced, becoming comparable to that of the bright one. We have thus demonstrated that applying a tilted exchange field is a highly efficient approach for dark-exciton brightening. It is worth noting that our theoretical prediction for $\theta=90^{\circ}$ is in good agreement with recent experiment data (see Fig. $2 \mathrm{a}$ of ref. ${ }^{41}$ ), which validates our theoretical results.

Finally, it is worth noting that the model we propose here can be straightforwardly extended to other TMD monolayers. We expect that Mo-based TMDs will show similar behavior while Wbased ones are very different. Opposite to $\mathrm{MoS}_{2}$, the dark exciton is the ground state for W-based monolayer TMDs. In this case, the dark exciton already has a larger population, especially at low temperatures, and thus an in-plane exchange field is more efficient in brightening the dark exciton ${ }^{25,39}$. The bright nature 

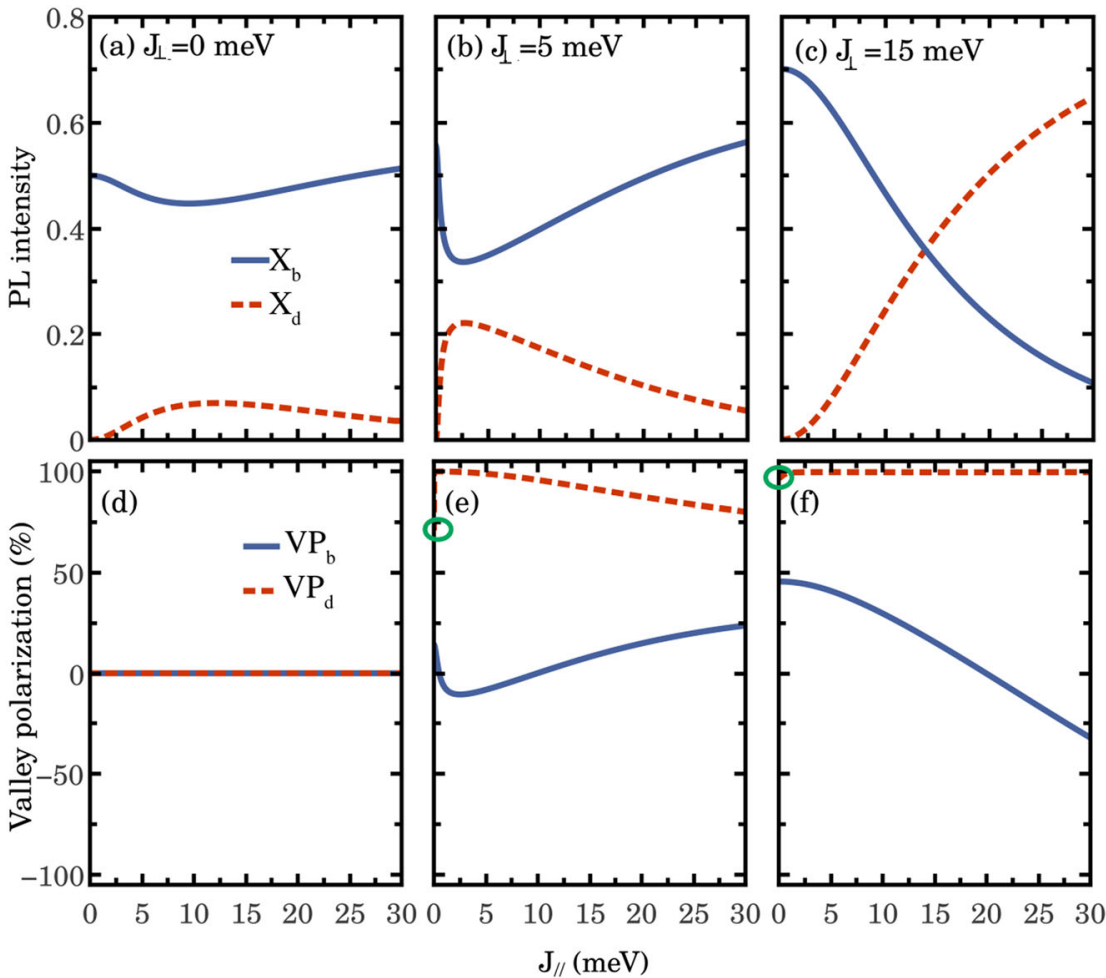

Fig. 4 Room-temperature PL intensity and exciton valley polarization. a-c PL intensity of bright $X_{b}$ and initially dark $X_{d}$ excitons in $\mathrm{K}$ valley as a function of $J_{\|}$for different values of $J_{\perp}$. $\mathbf{d}$ - $\mathbf{f}$ Valley polarization for bright and dark states. Green circles in $\mathbf{e}$ and $\mathbf{f}$ indicate that there are vanishing brightened-dark excitons at $J_{\|}=0$, at which the valley polarization of dark excitons is not well defined.

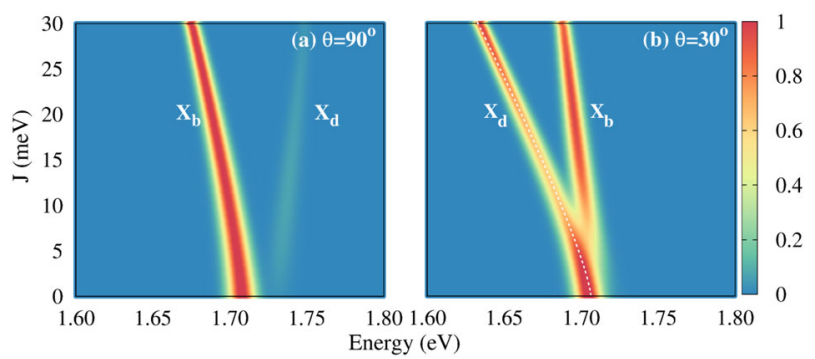

Fig. 5 Color map of the evolution of PL spectra as a function of the exchange field. Panel (a) dysplays in-plane $\left(\theta=90^{\circ}\right)$ and panel (b) shows tilted $\left(\theta=30^{\circ}\right)$ exchange field. The horizontal axis is the emission energy, and the vertical axis is the magnitude of the total exchange field. The PL intensity is normalized by the $X_{b}$ intensity at $J=0$.

of the ground state exciton in Mo-based monolayer, on the other hand, makes this types of materials more interesting for the gate control of the valley exciton emission via a tilted exchange field.

\section{DISCUSSION}

From the results above, it is clear that $J_{\perp}$ can effectively control the dark-exciton brightening analogous to a gate voltage tuning the source-drain current in a transistor. To demonstrate the performance of such magnetic gating, the intensity ON/OFF ratio $\left(I_{\mathrm{ON}} / I_{\mathrm{OFF}}\right)$ is plotted in the logarithmic scale (Fig. 6), as a function of $J_{\perp}$, at different $J_{\|}$values ranging from 0.1 to $10 \mathrm{meV}$. $I_{\mathrm{ON}} / l_{\mathrm{OFF}}$ is defined as the ratio of the emission intensity of the brighteneddark exciton at finite and zero $J_{\perp}$. It can be seen that for all $J_{\|}$values, $I_{\text {ON }} / I_{\text {OFF }}$ increases rapidly with increasing $J_{\perp}$, reaching maximum at the critical field $J_{\perp c}^{e x c}=5.1 \mathrm{meV}$, then decreases slowly with further increasing $J_{\perp}$. As discussed earlier, the induced spin

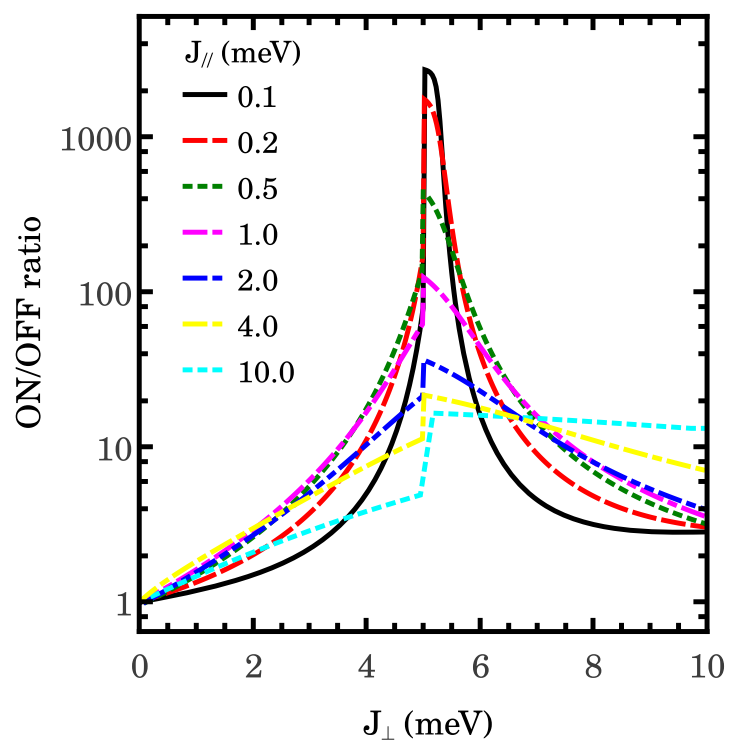

Fig. 6 The ON/OFF ratio of the brightened-dark-exciton emission of monolayer $\mathrm{MoS}_{2}$ subjected to a titled exchange field. The ON/ OFF ratio is plotted in logarithmic scale as a function of $J_{\perp}$, at different values of $J_{\|}$.

mixing is most effective at the bright-dark crossover point. The gating effect is more pronounced at smaller $J_{\|}$values. For example, the maximum ON/OFF ratio is found to be 2700 at $J_{\perp}=0.1 \mathrm{meV}$. Thus $\mathrm{MoS}_{2}$ monolayer emission can be gated effectively by an exchange field at room temperature, acting as a valley exciton transistor.

In conclusion, we proposed a theoretical design of magneticgateable valley exciton transistor, consisting of monolayer $\mathrm{MoS}_{2}$ 
subjected to a titled exchange field from a magnetic substrate. The in-plane and out-of-plane exchange fields work synergistically to brighten the originally spin-forbidden dark exciton. While the in-plane field mixes the spin-up and spin-down states, relaxing the optical selection rule; the out-of-plane field closes the gap between these states, increasing the dark-exciton population and thus greatly enhancing the emission intensity. The darkexciton brightening efficiency far exceeds that obtained by an inplane field. The brightened-dark-exciton emission possesses long lifetime and near $100 \%$ valley and spin polarization, controllable by an exchange field. This scheme can be used to engineer a magnetic-gateable valley exciton transistor with very large ON/ OFF ratio at room temperature, which can be useful for potential quantum information processing applications.

\section{METHODS}

Eleven bands tight-binding model

The effect of the tilted exchange field on the band structure of the monolayer $\mathrm{MoS}_{2}$ is described through an eleven bands tight-binding (TB) model which accurately describe the electronic structure of the $\mathrm{MoS}_{2}$ monolayer ${ }^{42}$. A top view of the monolayer and the first Brillouin zone are shown in Supplementary Figure 1a and c, respectively. The TB parameters are obtained by fitting from density functional theory calculation, as shown in Supplementary Figure $1 \mathrm{~b}$.

The total TB Hamiltonian is given by

$H_{T B}(\vec{k})=I_{2} \otimes H_{0}(\vec{k})+H_{S O}+H_{J}$.

The first term $I_{2} \otimes H_{0}(\vec{k})$ is spin-independent, and describes the local energy and the hopping up to second-nearest-neighbor terms between electrons in different atomic orbitals ${ }^{42} \cdot I_{2}$ is a $2 \times 2$ identity matrix in spin space. The second term of Eq. (1), $H_{\text {so }}=\sum_{a}\left(\lambda_{a} / \hbar\right) \overrightarrow{L_{a}} \cdot \overrightarrow{S_{a}}$, corresponds to the spin-orbit coupling Hamiltonian, where $\lambda_{a}$ is the intrinsic spin-orbit coupling strength, $\vec{L}_{a}$ is the atomic orbital angular momentum operator, and $\overrightarrow{S_{a}}$ is the electronic spin operator for the $a$ atom ( $a=$ Mo or S). The coupling constants $\lambda_{M o}=-0.0806 \mathrm{eV}$ and $\lambda_{S}=-0.0536 \mathrm{eV}$ are adjusted to fit the valence band (VB) spin splitting at the $\mathrm{K}$ and $\mathrm{K}^{\prime}$ points obtained by DFT calculation. Finally, the last term of Eq. (1) describes the effective exchange field driven by the coupling between the $\mathrm{MoS}_{2}$ monolayer and the ferromagnetic substrate:

$$
\begin{aligned}
H_{J}= & J\left(\sum_{\vec{R}_{l, l, l}, s, s^{\prime}}\left[\hat{m} \cdot \vec{S}_{s, s^{\prime}}\right] C_{\vec{R}, s, l}^{\dagger} C_{\vec{R}_{, s^{\prime}, l^{\prime}}} \delta_{l, l^{\prime}}\right. \\
& \left.+\frac{g_{L}}{g_{s}} \sum_{\vec{R}_{l, l, l}, s, s^{\prime}}\left[\hat{m} \cdot \vec{L}_{l, l^{\prime}}\right] C_{\vec{R}_{, s, l}^{\dagger}} C_{\vec{R}_{, s^{\prime}, l^{\prime}}} \delta_{s, s^{\prime}}\right)
\end{aligned}
$$

where $J$ is the effective exchange interaction strength, $g_{s}=2$ and $g_{L}=1$ are the spin and orbital $g$ factors of an electron, $\hat{m}$ is a unit vector that indicates the direction of the magnetization, $\vec{S}=\left(S_{x}, S_{y}, S_{z}\right)$ and $\vec{L}=$ $\left(L_{x}, L_{y}, L_{z}\right)$ are the Pauli spin matrices and the orbital angular momentum operator. Finally, $C_{\vec{R}, s, l}^{\dagger}\left(C_{\vec{R}_{s, l}}\right)$ is the electron creation (annihilation) operator for a spin $s$ and orbital angular momentum / state at the unit cell $R$. The first and the second terms in Eq. (2) describe the exchange interactions of electron spin and orbital angular momenta with the exchange field, respectively.

In general, the single-particle eigenfunctions are labeled by the indices of the energy bands $n$ and the Bloch wave number $\vec{k}$ and are expanded as a linear combination of atomic orbitals

$$
|n, \vec{k}\rangle=\sum_{\vec{R}, s l} C_{\vec{R}_{, s, l}}(n, \vec{k}) e^{i \vec{k} \cdot \vec{R}}|\vec{R}, s, I\rangle,
$$

where $\vec{R}$ describes the position of the unit cell and $|\vec{R}, s, I\rangle$ are the basis states.

The spin-orbit coupling lifts the spin degeneracy of the bands, leading to a spin-orbit splitting around $\mathrm{K}$ and $\mathrm{K}^{\prime}$ valleys, of the order of hundreds of $\mathrm{meV}$ in the valence bands (VBs) and tens of $\mathrm{meV}$ in the conduction bands (CBs). In this context, the value of the spin in $\hat{z}$ direction can also be used to label the band state:

$$
|n, \vec{k}, s\rangle=\sum_{\vec{R}, l} C_{\vec{R}_{, s, l}}(n, \vec{k}) e^{i \vec{k} \cdot \vec{R}}|\vec{R}, s, I\rangle,
$$

in such a way that

$$
\left\langle\hat{S}_{z}\right\rangle_{n, \vec{k}, s}=\left\langle n, \vec{k}, s\left|\hat{S}_{z}\right| n, \vec{k}, s\right\rangle= \pm 1 / 2
$$

The exchange term, Eq. (2), have different effects on the single-particle band structure depending on the direction of the magnetization. Specifically, for $\hat{m}=\hat{z}$, the bands are shifted, but $s$ is still a good quantum number. For a tilted magnetization, on the other hand, the spins are mixed:

$$
\left\langle\hat{S}_{z}\right\rangle_{n, \vec{k}}=\left\langle n, \vec{k}\left|\hat{S}_{z}\right| n, \vec{k}\right\rangle=\frac{1}{2}\left|c_{\uparrow}(n, \vec{k})\right|^{2}-\frac{1}{2}\left|c_{\downarrow}(n, \vec{k})\right|^{2}
$$

with

$$
\left|c_{\uparrow}(n, \vec{k})\right|^{2}=\sum_{\vec{R}, l} C_{\vec{R}, \uparrow, l}^{\dagger}(n, \vec{k}) C_{\vec{R}, \uparrow, l}(n, \vec{k})
$$

and

$$
\left|c_{\downarrow}(n, \vec{k})\right|^{2}=\sum_{\vec{R}, l} C_{\vec{R}, \downarrow, l}^{\dagger}(n, \vec{k}) C_{\vec{R}, \downarrow, l}(n, \vec{k})
$$

\section{Exciton eigenproblem}

With the single-particle description in hand, we calculate the excitonic states by solving the two-particle problem via Beth-Salpeter equation (BSE). More specifically, the exciton Hamiltonian is composed of the electron $H_{e}$ and the hole $H_{h}$ single-particle Hamiltonians, including the exchange interaction terms, plus the Coulomb interaction $V_{\vec{R}}$ which binds
the electron-hole pairs

$H_{e x}=H_{e}+H_{h}+V_{\vec{R}}$.

To account for the finite width of the TMD layer and the spatial inhomogeneity of the dielectric screening environment, we adopt the interaction Coulomb potential in the Keldysh form ${ }^{43-47}$

$$
V_{\vec{R}}=-\frac{e^{2}}{8 \epsilon_{0} \epsilon_{d} r_{0}}\left[H_{0}\left(\frac{|\vec{R}|}{r_{0}}\right)-Y_{0}\left(\frac{|\vec{R}|}{r_{0}}\right)\right],
$$

where $H_{0}$ and $Y_{0}$ are the Struve and Bessel functions of the second kind, respectively. In this work, we consider that the monolayer sits on a substrate and is exposed to air, which leads to an effective dielectric constant given by $\epsilon_{d}=\left(\epsilon_{\text {sub }}+\epsilon_{\text {air }}\right) / 2$. Furthermore, $r_{0}$ represents a characteristic length defined by $r_{0}=2 \pi X_{2 D} / \epsilon_{d}$, with $X_{2 D}$ being the twodimensional polarizability. Throughout this work, we consider $\epsilon_{d}=2.5$ and $X_{2 D}=6.60 \AA^{47}$ for the $M_{0} S_{2}$ monolayer subjected to a magnetic substrate.

Since we study periodic lattices, it is more convenient to work in the reciprocal space; in $k$-space, the Keldysh potential acquires the form ${ }^{48,49}$

$$
v_{\vec{Q}}=-\frac{e^{2}}{2 \epsilon_{0} \epsilon_{d}|\vec{Q}|\left(1+r_{0}|\vec{Q}|\right)},
$$

where $\vec{Q}$ is the exciton momentum, defined as the difference between the electron and the hole momenta, i.e., $\vec{Q}=\vec{k}-\vec{k}^{\prime}$.

The exciton wave function is defined as a linear combination of singleparticle electron-hole pairs,

$$
\Psi_{e x}^{M}(\vec{Q})=\sum_{c, v, \vec{k}} A^{M}, \vec{v}, \vec{k}, \vec{Q}(|c, \vec{k}+\vec{Q}\rangle \otimes|v, \vec{k}\rangle),
$$

where the indices $c$ and $v$ refer to the conduction and valence states, with momentum $\vec{k}+\vec{Q}$ and $\vec{k}$, respectively. Since we are interested in the low-energy excitons, we consider a four bands model, that is, $n=\left\{c_{1}, c_{2}, v_{1}\right.$, $v_{2}$ \}. The exciton eigenproblem on the basis displayed in Eq. (12) leads to the following BSE

$$
\begin{gathered}
\left(E_{c, \vec{k}+\vec{Q}}-E_{v, \vec{k}}\right) A_{c, v, \vec{k}, \vec{Q}}^{M}+\frac{1}{s} \sum_{k^{\prime}, v^{\prime}, c^{\prime}} W_{(\vec{k}, v, c),\left(\vec{k}^{\prime}, v^{\prime}, c^{\prime}\right),} \vec{Q}_{c^{\prime}, v^{\prime}, \vec{k}^{\prime}, \vec{Q}}^{M} \\
=E_{\vec{Q}}^{M} A_{c, v, \vec{k}, \vec{Q}}^{M}
\end{gathered}
$$


In the equation above, $S=S_{c} N_{k}^{2}$ is the total area of the crystal, where $S_{c}=\sqrt{3} a^{2} / 2$ and $a$ is the lattice constant. Furthermore, $W_{(\vec{k}, v, c),\left(\vec{k}^{\prime}, v^{\prime}, c^{\prime}\right)}, \vec{Q}$ represents the matrix element of the many-body Coulomb potential including direct $W^{d}$ and exchange $W^{x}$ terms, and $E_{\vec{Q}}^{M}$ is the energy of the Mth excitonic state with momentum $\vec{Q}$.

To simplify our calculation, we apply the Tamm-Dancoff approximation (TDA) to the many-body Coulomb potential, which neglects the orbital character of the Coulomb interaction. In this case $W_{(\vec{k}, v, c),\left(\vec{k}^{\prime}, v^{\prime}, c^{\prime}\right), \vec{Q}}^{d}$ and $W_{(\vec{k}, v, c),\left(\vec{k}^{\prime}, v^{\prime}, c^{\prime}\right), \vec{Q}}$ are given by

$W_{(\vec{k}, v, c),\left(\overrightarrow{\left.k^{\prime}, v^{\prime}, c^{\prime}\right),}\right.}^{d} \vec{Q}=V_{\vec{k}-\overrightarrow{k^{\prime}}}\left\langle c, \vec{k}+\vec{Q} \mid c^{\prime}, \overrightarrow{k^{\prime}}+\vec{Q}\right\rangle\left\langle v, \vec{k} \mid v^{\prime}, \overrightarrow{k^{\prime}}\right\rangle$

and

$W_{\left(\vec{k}_{, v, c),\left(\vec{k}^{\prime}, v^{\prime}, c^{\prime}\right),}^{x} \vec{Q}\right.}=-v_{\vec{Q}}\langle c, \vec{k}+\vec{Q} \mid v, \vec{k}\rangle\left\langle v^{\prime}, \overrightarrow{k^{\prime}} \mid c^{\prime}, \overrightarrow{k^{\prime}}+\vec{Q}\right\rangle$.

Although a complete exciton band structure includes excitons with nonzero center of mass momentum, we focus our attention only on optical vertical transitions close to $\mathrm{K}$ and $\mathrm{K}^{\prime}$ point, i.e., $\vec{Q}=0$. For $\vec{Q}=0$, the exchange term $W_{(\vec{k}, v, c),\left(\vec{k}^{\prime}, v^{\prime}, c^{\prime}\right), \vec{Q}}$ vanishes due to orthogonal properties of $\langle c, \vec{k}+\vec{Q} \mid v, \vec{k}\rangle$ and $\left\langle v^{\prime}, \overrightarrow{k^{\prime}} \mid c^{\prime}, \overrightarrow{k^{\prime}}+\vec{Q}\right\rangle^{45}$.

\section{Exciton absorption spectra}

The exciton absorption intensity $A(\omega)$ is given by Fermi's golden rule

$A(\omega)=\frac{e^{2} \pi}{\hbar^{2} \omega V_{c}} \sum_{M} O_{M} \delta\left(\hbar \omega-E_{0}^{M}\right)$,

where $E_{0}^{M}$ is the energy of the $M$ th excitonic state with $\vec{Q}=0$ and $O_{M}$ is the oscillator strength, defined by

$O_{M}=\left|\sum_{\vec{k}, c, v} A_{c, v, \vec{k}, 0}^{M}\left\langle c, \vec{k}\left|H_{L M}(\vec{k})\right| v, \vec{k}\right\rangle\right|^{2}$,

where $H_{L M}=\frac{\partial H_{T B}}{\partial k_{x}}$ represents the light-matter coupling for linear light polarization.

The value of $O_{M}$ defines the optical activity of the excitonic state and depends on the electron and hole spins.

Energy shift and brightening of exciton states via magnetic proximity effect

Generally, the electron-hole Coulomb interaction couples multiple $C B$ and VB states. The lower energy states at the $\Gamma$ point $(\vec{Q}=0)$, however, can be properly identified as the strongly localized $A$ and $B$ excitons ${ }^{50}$. Such states are formed at the direct band gaps ( $K$ and $K^{\prime}$ points) with the holes and electrons placed in a well-defined CB and VB. Specifically, A excitons involve electronic transitions between the highest valence band $\left(v_{1}\right)$ and the two lowest spin-split conduction bands $\left(c_{1}\right.$ and $\left.c_{2}\right)$ in both $\mathrm{K}$

$\left|X_{1}^{K}\right\rangle \approx\left|c_{1}, s_{c 1}\right\rangle \otimes\left|v_{1}, s_{v 1}\right\rangle$

$\left|X_{2}^{K}\right\rangle \approx\left|c_{2}, s_{c 2}\right\rangle \otimes\left|v_{1}, s_{v 1}\right\rangle$

and $\mathrm{K}^{\prime}$ valley

$\left|X_{1}^{K^{\prime}}\right\rangle \approx\left|c_{1}^{\prime}, s_{c 1}^{\prime}\right\rangle \otimes\left|v_{1}^{\prime}, s_{v 1}^{\prime}\right\rangle$

$\left|X_{2}^{K^{\prime}}\right\rangle \approx\left|c_{2}^{\prime}, s_{c 2}^{\prime}\right\rangle \otimes\left|v_{1}^{\prime}, s_{v 1}^{\prime}\right\rangle$,

with $\left|n, s_{n}\right\rangle\left(n=\left\{c_{1}, c_{2}, v_{1}\right\}\right)$ the single-particle state (Eq. (4) with fixed $\vec{k}$ around the valleys).

Furthermore, in the absence of an in-plane exchange field, we have seen that the spin of the CB and VB are well defined. Therefore, A excitons can also be classified as bright $\left(X_{b}\right.$, spin allowed, parallel spins in both bands, i.e., $\left.s_{c i}=s_{v 1}\right)$ and dark ( $X_{d}$, spin forbidden, antiparallel spin configuration, $s_{c i}$ $=-s_{v 1}$ ) states depending on the spin configuration of the singleparticle bands.

An out-of-plane exchange field maintains the spin quantum numbers; therefore, the oscillator strength of the excitons is hardly affected. However, it decreases the energy separation between bright and dark states in the $\mathrm{K}$ valley, while increases the intravalley energy separation in $\mathrm{K}^{\prime}$ valley. Since the spins of the conduction and valence bands are well defined, each excitons is fully bright or fully dark. The in-plane field component, on the other hand, tilts the spins, in such a way that the excitons can be described as linear combinations of bright $\left|X_{b}\right\rangle$ and dark $\left|X_{d}\right\rangle$ components:

$$
\begin{aligned}
\left|X_{1}^{K}\right\rangle & =a_{1 B}\left|X_{b}\right\rangle+a_{1 D}\left|X_{d}\right\rangle \\
\left|X_{2}^{K}\right\rangle & =a_{2 B}\left|X_{b}\right\rangle+a_{2 D}\left|X_{d}\right\rangle \\
\left|X_{1}^{K^{\prime}}\right\rangle & =a_{1 B}^{\prime}\left|X_{b}\right\rangle+a_{1 D}^{\prime}\left|X_{d}\right\rangle \\
\left|X_{2}^{K^{\prime}}\right\rangle & =a_{2 B}^{\prime}\left|X_{b}\right\rangle+a_{2 D}^{\prime}\left|X_{d}\right\rangle,
\end{aligned}
$$

where the coefficients $a_{i j}\left(i=\{1,2\}\right.$ and $\left.\tau=\left\{K, K^{\prime}\right\}\right)$ depend on the singleparticle spin components, namely,

$\left|a_{i B}^{\tau}\right|^{2}=\frac{1+4\left(\left\langle\hat{S}_{z}\right\rangle_{v_{1}, \tau}\left\langle\hat{S}_{z}\right\rangle_{C_{i}, \tau}\right)}{2}$

and

$\left|a_{i D}^{\tau}\right|^{2}=\frac{1-4\left(\left\langle\hat{S}_{z}\right\rangle_{v_{1}, \tau}\left\langle\hat{S}_{z}\right\rangle_{c_{i}, \tau}\right)}{2}$

Above, $\left\langle\hat{S}_{z}\right\rangle_{n, \tau}$ is the spin expectation value of the $n$-th band at the corners of the hexagonal Brillouin zone, as defined in Eqs. (6)-(8). Note that, in the absence of in-plane exchange field, we have $\left\langle\hat{S}_{z}\right\rangle_{y_{1}, T}\left\langle\hat{S}_{z}\right\rangle_{c_{i}, T}=1 / 4(-1 / 4)$ which leads to $\left|a_{i B}^{T}\right|=1\left(\left|a_{i B}^{T}\right|=0\right)$ and $\left|a_{i D}^{\tau}\right|=0\left(\left|a_{i D}^{T}\right|=1\right)$ for bright (dark) excitons. With an in-plane magnetic field, $\left\langle\hat{S}_{z}\right\rangle_{c_{i},}$ can assume different values from $-1 / 2$ to $1 / 2$, depending on the exchange field amplitude and direction. Therefore, $\left|a_{i B}^{\tau}\right|$ and $\left|a_{i D}^{\tau}\right|$ acquire different values from 0 to 1 . The bright and dark-exciton energies in $K$ and $K^{\prime}$ valleys are shown in Supplementary Figure 3 for $J=25 \mathrm{meV}$, pointing in different directions. The values without exchange field are also shown, for comparison.

\section{Exciton dynamics}

The BSE description of the exciton eigenproblem provides the eigenfunction, eigenvalues and the absorption spectra of the exciton states, including the effect of the exchange field on these properties. The exciton emission, however, involves Coulomb and phonon mediated intra- and intervalley scatterings between different quasi-particles. We propose a theoretical framework that uses the outputs of the BSE calculation as inputs to an effective description of the exciton dynamics. More specifically, we consider the evolution of the energy and spin components of the four lower exciton states described in the section above and investigate the $\mathrm{PL}$ intensity and the valley polarization via the exciton recombination kinetics, given by a set of four coupled rate equations, We include the valley-selective brightening of dark excitons, scatterings between intravalley A excitons, as well as intervalley scattering between bright components, as described by the following equation of motion

$\dot{n}_{i}^{\tau}=\left|a_{i B}^{T}\right|^{2} g-\left(\Gamma_{i i}^{T T}+\Gamma_{i j}^{T T}+\left|a_{i B}^{\tau}\right|^{2} \sum_{l=1}^{2}\left|a_{l B}^{\tau^{\prime}}\right|^{2} \Gamma_{i l}^{T^{\prime}}\right) n_{i}^{\tau}+\Gamma_{j i}^{T T} n_{j}^{\tau}+\left|a_{i B}^{\tau}\right|^{2} \sum_{l=1}^{2}\left|a_{l B}^{\tau^{\prime}}\right|^{2} \Gamma_{l i}^{\tau^{\prime} \tau} n_{l}^{\tau^{\prime}}$,

where $n_{i}^{\tau}$ is the density of state $i=\{1,2\}$ in the $\tau=\left\{K, K^{\prime}\right\}$ valley, and $\dot{n}$ represents the time derivative; the general formula of Eq. (23) represents the time evolution of the four states, $X_{1}^{K}, X_{2}^{K}, X_{1}^{K^{\prime}}$, and $X_{2}^{K^{\prime}}$. The first term on the right hand side corresponds to the photocreation rate $g$ of the state, weighted by its bright component. The recombination rate, $\Gamma_{i i}^{\tau \tau}$ is a linear combination of the bright and dark recombination rates: $\Gamma_{i i}^{T \tau}=\left|a_{i B}^{\tau}\right|^{2} \Gamma_{b}+\left|a_{i d}^{\tau}\right|^{2} \Gamma_{d}$, with $\Gamma_{b}=(10 \mathrm{ps})^{-151}$ and $\Gamma_{d}=(1 \mathrm{~ns})^{-152}$, for radiative and non-radiative recombination rates, respectively.

The intravalley scattering rate, $\Gamma_{i j}^{T \tau}$, is taken to be $0.1 \mathrm{ps}^{-153}$ for $i>j$ (scattering from the excited to the ground state) and $u^{\tau}(T, J) 0.1 \mathrm{ps}^{-1}$ for $i<j$, i.e., the scattering from ground to excited excitonic state depends on the Boltzmann distribution $u^{T}(T, J)=\exp \left(-\left|\Delta \mathrm{E}^{T}(J)\right| / k_{b} T\right)$, reflecting the presence of the valley- and exchange-field-dependent energy barrier $\Delta E^{\tau}$ between the two sates in $\tau$ valley (see Supplementary Figure 4 for the energy difference as a function of exchange). 
Finally, we consider the intervalley scattering between bright components, given by

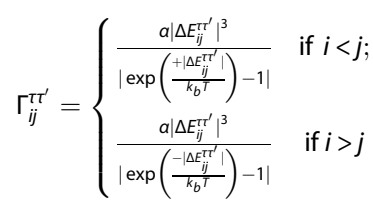

which describes the emission or absorption of a phonon ${ }^{54}$ with energy correspondent to the valley splitting $\Delta E_{i j}^{\tau^{\prime}}$. Furthermore, $a$ is the exciton-phonon coupling strength. Throughout this work we consider $a$ $=10^{5} \mathrm{ps}^{-1} \mathrm{eV}^{-3}$, so intra- and intervalley scattering rates are of the same order for the exchange field strengths analyzed here. Even though there are mechanisms which can cause intervalley scattering between dark states, experimental results reveal that such quasi-particles have much longer valley lifetime; the relevant scattering time is about one order of magnitude larger than that of bright states ${ }^{55}$. Thus, the intervalley scattering between two dark components is not considered. Results presented in the main text were obtained for the steady state, where the density of each state is obtained by letting the left hand side of Eq. (23) equal to zero. The PL intensity is then given by $I_{i}^{\tau}=\left|a_{i, B}\right|^{2} \Gamma_{i i}^{T \tau} n_{i}^{\tau}$. Furthermore, the valley polarization is calculated by comparing the intensity of corresponding states in $\mathrm{K}$ and $\mathrm{K}^{\prime}$ valleys: $V P_{i}=\frac{l_{i}^{K}-l_{i}^{K^{\prime}}}{l_{i}^{K}+l_{i}^{K^{\prime}}}$.

\section{Reporting summary}

Further information on research design is available in the Nature Research Reporting Summary linked to this article.

\section{DATA AVAILABILITY}

The data that support the findings of this study are available from the corresponding author upon reasonable request.

\section{CODE AVAILABILITY}

The numerical codes for single-particle band structure, exciton eigenproblem, and exciton dynamics are available from the corresponding author upon reasonable request.

Received: 25 November 2019; Accepted: 20 May 2020; Published online: 07 July 2020

\section{REFERENCES}

1. Choi, W. et al. Recent development of two-dimensional transition metal dichalcogenides and their applications. Mater. Today 20, 116-130 (2017).

2. Roldán, R. et al. Theory of $2 \mathrm{~d}$ crystals: graphene and beyond. Chem. Soc. Rev. 46, 4387-4399 (2017).

3. Liu, G.-B. et al. Electronic structures and theoretical modelling of two-dimensional group-VIB transition metal dichalcogenides. Chem. Soc. Rev. 44, 2643-2663 (2015).

4. Wang, Q. H. et al. Electronics and optoelectronics of two-dimensional transition metal dichalcogenides. Nat. Nanotechnol. 7, 699 (2012).

5. Bernardi, M., Ataca, C., Palummo, M. \& Grossman, J. C. Optical and electronic properties of two-dimensional layered materials. Nanophotonics 6, 479-493 (2017).

6. Mak, K. F., Lee, C., Hone, J., Shan, J. \& Heinz, T. F. Atomically thin $\operatorname{MoS}_{2}$ : a new direct-gap semiconductor. Phys. Rev. Lett. 105, 136805 (2010).

7. Mouri, S. et al. Nonlinear photoluminescence in atomically thin layered $\mathrm{WSe}_{2}$ arising from diffusion-assisted exciton-exciton annihilation. Phys. Rev. B 90, 155449 (2014).

8. Dias, A. C., Fu, J. Y., Lelovsky, L. V. \& Qu, F. Y. Robust effective Zeeman energy in monolayer $\mathrm{MoS}_{2}$ quantum dots. J. Phys. Condens. Matter 28, 375803 (2016).

9. Wu, F., Qu, F. \& MacDonald, A. H. Exciton band structure of monolayer MoS 2 . Phys. Rev. B 91, 075310 (2015).

10. Robert, $C$. et al. Exciton radiative lifetime in transition metal dichalcogenide monolayers. Phys. Rev. B 93, 205423 (2016).

11. Plechinger, G. et al. Trion fine structure and coupled spin-valley dynamics in monolayer tungsten disulfide. Nat. Commun. 7, 12715 (2016).
12. Godde, T. et al. Exciton and trion dynamics in atomically thin $\mathrm{MoSe}_{2}$ and $\mathrm{WSe}_{2}$ : effect of localization. Phys. Rev. B 94, 165301 (2016).

13. Gao, F., Gong, Y. J., Titze, M., Almeida, R., Ajayan, P. M. \& Li, H. B. Valley trion dynamics in monolayer $\mathrm{MoSe}_{2}$. Phys. Rev. B 94, 245413 (2016).

14. Mak, K. F. et al. Tightly bound trions in monolayer $\mathrm{MoS}_{2}$. Nat. Mater. 12, 207 (2013).

15. Zhu, Z., Cheng, Y. \& Schwingenschlögl, U. Giant spin-orbit-induced spin splitting in two-dimensional transition-metal dichalcogenide semiconductors. Phys. Rev. $B$ 84, 153402 (2011).

16. Kośmider, K., González, J. W. \& Fernández-Rossier, J. Large spin splitting in the conduction band of transition metal dichalcogenide monolayers. Phys. Rev. B 88, 245436 (2013).

17. Malic, E. et al. Dark excitons in transition metal dichalcogenides. Phys. Rev. Mater. 2, 014002 (2018).

18. Yu, H., Liu, G.-B., Gong, P., Xu, X. \& Wang, Y. Dirac cones and Dirac saddle points of bright excitons in monolayer transition metal dichalcogenides, Nature Commun. 5, 3876 (2014).

19. Zhu, C. et al. Exciton valley dynamics probed by Kerr rotation in $\mathrm{WSe}_{2}$ monolayers. Phys. Rev. B 90, 161302 (2014).

20. Jones, A. M. et al. Optical generation of excitonic valley coherence in monolayer WSe $e_{2}$ Nat. Nanotechnol. 8, 634 (2013).

21. $\mathrm{Yu}, \mathrm{T}$. \& Wu, M. Valley depolarization due to intervalley and intravalley electronhole exchange interactions in monolayer $\mathrm{MoS}_{2}$. Phys. Rev. B 89, 205303 (2014).

22. Glazov, M. et al. Exciton fine structure and spin decoherence in monolayers of transition metal dichalcogenides. Phys. Rev. B 89, 201302 (2014).

23. Robert, $C$. et al. Fine structure and lifetime of dark excitons in transition metal dichalcogenide monolayers. Phys. Rev. B 96, 155423 (2017).

24. Poem, E. et al. In Quantum Electronics and Laser Science Conference QPDA9 (Optical Society of America, 2010).

25. Zhang, X.-X. et al. Magnetic brightening and control of dark excitons in monolayer WSe $\mathrm{W}_{2}$. Nat. Nanotechnol. 12, 883 (2017).

26. Molas, M. et al. Brightening of dark excitons in monolayers of semiconducting transition metal dichalcogenides. 2D Mater. 4, 021003 (2017).

27. Ye, Z. et al. Probing excitonic dark states in single-layer tungsten disulphide. Nature 513, 214 (2014).

28. Wang, G. et al. In-plane propagation of light in transition metal dichalcogenide monolayers: optical selection rules. Phys. Rev. Lett. 119, 047401 (2017).

29. Zhou, Y. et al. Probing dark excitons in atomically thin semiconductors via nearfield coupling to surface plasmon polaritons. Nat. Nanotechnol. 12, 856 (2017).

30. Park, K.-D. et al. Radiative control of dark excitons at room temperature by nanooptical antenna-tip purcell effect. Nat. Nanotechnol. 13, 59 (2018).

31. Zhang, X.-X., You, Y., Zhao, S. Y. F. \& Heinz, T. F. Experimental evidence for dark excitons in monolayer wse 2. Phys. Rev. Lett. 115, 257403 (2015).

32. Zhao, C. et al. Enhanced valley splitting in monolayer $\mathrm{WSe}_{2}$ due to magnetic exchange field. Nat. Nanotechnol. 12, 757 (2017).

33. Norden, $\mathrm{T}$. et al. Giant valley splitting in monolayer $\mathrm{WS}_{2}$ by magnetic proximity effect. Nat. Commun. 10, 4163 (2019).

34. Zhong, D. et al. Van der Waals engineering of ferromagnetic semiconductor heterostructures for spin and valleytronics. Sci. Adv. 3, e1603113 (2017).

35. $\mathrm{Xu}, \mathrm{L}$. et al. Large valley splitting in monolayer $\mathrm{WS}_{2}$ by proximity coupling to an insulating antiferromagnetic substrate. Phys. Rev. B 97, 041405 (2018).

36. Li, Q., Chen, K.-Q. \& Tang, L.-M. Large valley splitting in van der Waals heterostructures with type-iii band alignment. Phys. Rev. Appl. 13, 014064 (2020).

37. Scharf, B., Xu, G., Matos-Abiague, A. \& Žutić, I. Magnetic proximity effects in transition-metal dichalcogenides: converting excitons. Phys. Rev. Lett. 119, 127403 (2017).

38. Unuchek, D. et al. Room-temperature electrical control of exciton flux in a van der Waals heterostructure. Nature 560, 340 (2018).

39. Vasconcelos, R., Bragança, H., Qu, F. \& Fu, J. Dark exciton brightening and its engaged valley dynamics in monolayer WSe 2 . Phys. Rev. B 98, 195302 (2018).

40. Qu, F. et al. Controlling valley splitting and polarization of dark-and bi-excitons in monolayer $W_{2}$ by a tilted magnetic field. 2D Mater. 6, 045014 (2019).

41. Lu, Z. et al. Magnetic field mixing and splitting of bright and dark excitons in monolayer $\mathrm{MoSe}_{2}$. 2D Mater. 7, 015017 (2019).

42. Dias, A. C., Qu, F., Azevedo, D. L. \& Fu, J. Band structure of monolayer transitionmetal dichalcogenides and topological properties of their nanoribbons: nextnearest-neighbor hopping. Phys. Rev. B 98, 075202 (2018).

43. Keldysh, L. V. Coulomb interaction in thin semiconductor and semimetal films. JETP Lett. 29, 658 (1979).

44. Cudazzo, P., Tokatly, I. V. \& Rubio, A. Dielectric screening in two-dimensional insulators: Implications for excitonic and impurity states in graphane. Phys. Rev. B 84, 085406 (2011).

45. Wu, F., Qu, F. \& MacDonald, A. H. Exciton band structure of monolayer MoS 2 . Phys. Rev. B 91, 075310 (2015). 
46. Ridolfi, E., Lewenkopf, C. H. \& Pereira, V. M. Excitonic structure of the optical conductivity in MoS 2 monolayers. Phys. Rev. B 97, 205409 (2018).

47. Berkelbach, T. C., Hybertsen, M. S. \& Reichman, D. R. Theory of neutral and charged excitons in monolayer transition metal dichalcogenides. Phys. Rev. B 88, 045318 (2013).

48. Chernikov, A. et al. Exciton binding energy and nonhydrogenic Rydberg series in monolayer $\mathrm{WS}_{2}$. Phys. Rev. Lett. 113, 076802 (2014).

49. Rodin, A. S., Carvalho, A. \& NetoCastro, A. H. Excitons in anisotropic twodimensional semiconducting crystals. Phys. Rev. B 90, 075429 (2014).

50. Ramasubramaniam, A. Large excitonic effects in monolayers of molybdenum and tungsten dichalcogenides. Phys. Rev. B 86, 115409 (2012).

51. You, Y. et al. Observation biexcitons monolayer WSe $\mathrm{W}_{2}$. Nat. Phys. 11, 477 (2015).

52. Palummo, M., Bernardi, M. \& Grossman, J. C. Exciton radiative lifetimes in twodimensional transition metal dichalcogenides. Nano Lett. 15, 2794-2800 (2015).

53. Baranowski, M. et al. Dark excitons and the elusive valley polarization in transition metal dichalcogenides. 2D Mater. 4, 025016 (2017).

54. Surrente, A. et al. Intervalley scattering of interlayer excitons in a $\mathrm{MoS}_{2} / \mathrm{MoSe}_{2} /$ $\mathrm{MoS}_{2}$ heterostructure in high magnetic field. Nano Lett. 18, 3994-4000 (2018).

55. Smoleński, T. et al. Tuning valley polarization in a $\mathrm{WSe}_{2}$ monolayer with a tiny magnetic field. Phys. Rev. X 6, 021024 (2016).

\section{ACKNOWLEDGEMENTS}

The authors are grateful to CENAPAD-SP for the provision of computational facilities. This work was partially supported by CNPq, FAPDF, Coordenação de Aperfeiçoamento de Pessoal de Nível Superior - Brasil (CAPES) - Finance Code 001, and US National Science Foundation (US NSF DMR-1104994 and CBET-1510121). The authors thank A. H. MacDonald and S.J. Xie for illuminating discussions.

\section{AUTHOR CONTRIBUTIONS}

F.Q. and H.Z. conceived the idea; A.C.D. built the TB and BSE numerical codes; H.B. performed the numerical calculation and designed the rate equations for exciton dynamics; J.H.C. calculated the effective model, F.Q., H.Z., and H.B wrote the paper. All the authors contributed to the interpretation of the results and commented on the paper.

\section{COMPETING INTERESTS}

The authors declare no competing interests.

\section{ADDITIONAL INFORMATION}

Supplementary information is available for this paper at https://doi.org/10.1038/ s41524-020-00356-w.

Correspondence and requests for materials should be addressed to H.Z. or F.Q.

Reprints and permission information is available at http://www.nature.com/ reprints

Publisher's note Springer Nature remains neutral with regard to jurisdictional claims in published maps and institutional affiliations.

Open Access This article is licensed under a Creative Commons Attribution 4.0 International License, which permits use, sharing, adaptation, distribution and reproduction in any medium or format, as long as you give appropriate credit to the original author(s) and the source, provide a link to the Creative Commons license, and indicate if changes were made. The images or other third party material in this article are included in the article's Creative Commons license, unless indicated otherwise in a credit line to the material. If material is not included in the article's Creative Commons license and your intended use is not permitted by statutory regulation or exceeds the permitted use, you will need to obtain permission directly from the copyright holder. To view a copy of this license, visit http://creativecommons. org/licenses/by/4.0/.

(c) The Author(s) 2020 